\title{
Abordaje nutricional de un paciente en cuidados paliativos. Análisis de un caso clínico
}

\author{
Nutritional approach of a patient in palliative care. Analysis of a clinical case \\ Abordagem nutricional de um paciente em cuidados paliativos. Análise de um caso clínico
}

\author{
Claudia Patricia Maza Moscoso ${ }^{1,2 *}$, Luis Demetrio González Patzán ${ }^{1,3}$, Miriam Sucely Corado López ${ }^{1}$
}

Recibido: 14 de noviembre 2020. Aceptado para publicación: 10 de diciembre 2020. https://doi.org/10.35454/rncm.v4n2.233

\section{Resumen}

La malnutrición en pacientes con cáncer gástrico es un problema frecuente que debe atenderse. La nutrición artificial y la hidratación al final de la vida se presentan como parte del tratamiento médico, cuidado o acto de humanidad. Se debe brindar soporte nutricional por vía oral, enteral o parenteral, según la condición clínica, e individualizando cada caso. El objetivo del presente caso clínico es describir el manejo nutricional de un paciente en cuidados paliativos, hospitalizado en una institución hospitalaria de tercer nivel en Ciudad de Guatemala. Se reporta el caso de un paciente masculino de 27 años con adenocarcinoma gástrico, el cual fue tratado con quimioterapia, pero recidivó y avanzó rápidamente. Se describen las diferentes fases del abordaje nutricional, comparando con lo que se indica en la literatura. La nutrición parenteral en el hospital continuó hasta el último día de su vida, debido a la obstrucción y al fuerte deseo del paciente y su familia de aferrarse a la vida. A los 11 meses del diagnóstico, el paciente fallece. La nutrición parenteral en casa hubiera sido la indicada en un caso como este; sin embargo, la mayoría de las instituciones hospitalarias guatemaltecas no brindan este servicio, por lo que el paciente debe costearla, lo que limita su acceso.

Palabras clave: cáncer gástrico, obstrucción intestinal, terapia nutricional, autonomía.

\section{Summary}

Malnutrition in patients with gastric cancer is a common problem that must be addressed. Artificial nutrition and hydration at the end-of-life are considered part of medical treatment, care process, or act of humanity. Oral, enteral, or parenteral nutritional support must be provided according to the clinical condition and on a case-by-case basis. The objective of this case study is to describe the nutritional management of a palliative care patient hospitalized in a third-level health care institution in Guatemala City.

The case of a 27-year-old male patient with gastric adenocarcinoma treated with chemotherapy, but who relapsed and progressed rapidly, is reported. The different phases of the nutritional approach used are described and compared to what is described in published literature. Parenteral nutrition in the hospital continued until the patient's death, due to the gastrointestinal obstruction and the strong desire of the patient and his family to hang on to life. The patient died 11 months after diagnosis. In cases like this home parenteral nutrition would have been indicated. However, most Guatemalan health care institutions do not provide this service, and moreover, the patient must pay for it, thereby limiting the use of this therapy at home.

Keywords: Gastric Cancer; Intestinal Obstruction; Nutritional Therapy; Autonomy.

\section{Resumo}

A desnutrição em pacientes com câncer gástrico é um problema comum que deve ser tratado. A nutrição artificial e a hidratação no final da vida são apresentadas como parte do tratamento médico, cuidado e / ou ato de humanidade.

O suporte nutricional deve ser fornecido por via oral, enteral e / ou parenteral, de acordo com o quadro clínico e individualizando cada caso. O objetivo deste caso clínico é descrever o manejo nutricional de um paciente em cuidados paliativos internado em uma Instituição Hospitalar de atenção terciaria na Cidade da Guatemala.

É relatado o caso de um paciente masculino de 27 anos de idade, com adenocarcinoma gástrico, que foi tratado com quimioterapia, mas apresentou recidiva e evoluiu rapidamente. São descritas as diferentes fases da abordagem nutricional utilizadas em comparação com o descrito na literatura.

A nutrição parenteral no hospital continuou até o último dia de sua vida, devido à obstrução e ao forte desejo do paciente e de sua família de o manterem vivo. Onze meses após o diagnóstico, o paciente foi a óbito. A nutrição parenteral em domicilio teria sido indicada em casos como este.

No entanto, a maioria das instituições hospitalares da Guatemala não dispõem desse serviço, de modo que o paciente deve pagar por ele, limitando o acesso.

Palavras-chave: câncer gástrico, obstrução intestinal, terapia nutricional, autonomia. 
Centro Médico Militar, Ciudad Guatemala, Guatemala.

2 Universidad del Valle de Guatemala. Ciudad de Guatemala. Guatemala

3 Hospital General San Juan de Dios, Ciudad de Guatemala. Guatemala
*Correspondencia: Claudia Patricia Maza Moscoso. cmaza34@hotmail.com

\section{INTRODUCCIÓN}

En 2018, el cáncer gástrico fue el quinto cáncer más frecuente en el mundo, con 1 millón de casos nuevos, lo que supone el 5,7\% del total de cánceres. Este es un cáncer común en Latinoamérica, Europa del Este y algunos países del Oriente Medio, y es menos frecuente en Europa, Estados Unidos, Australia y África. El riesgo de desarrollar un cáncer gástrico aumenta a partir de los 50 años, y es 2 veces más frecuente en varones que en mujeres ${ }^{(1)}$.

La terapia nutricional se debe considerar para todos los pacientes con cáncer que se encuentran en riesgo o están malnutridos, y en aquellos que están recibiendo un tratamiento que puede afectar su capacidad de ingerir alimentos y causar malnutrición ${ }^{(2,3)}$. Las guías de la European Society for Clinical Nutrition and Metabolism (ESPEN) sugieren que "la nutrición enteral debe ser provista con el objeto de minimizar la pérdida de peso, siempre y cuando el paciente esté de acuerdo y no haya iniciado la fase de la muerte"(4). Algunos pacientes con cáncer avanzado, especialmente aquellos con tumores incurables del tracto gastrointestinal superior, presentan alteración severa del estado nutricional, y pueden fallecer de inanición progresiva debido a la imposibilidad mecánica de la ingestión de alimentos, a la inflamación y al hipercatabolismo asociado con el tumor. Por esta razón se debe considerar la terapia nutricional a lo largo de los cuidados paliativos hospitalarios, y en algunos casos en casa ${ }^{(4)}$.

La terapia puede ser oral, enteral o parenteral. El objetivo de la terapia nutricional no debe ser prevenir el hambre y la sed. Los pacientes con cáncer avanzado generalmente se encuentran anoréxicos. Además, el hambre es un síntoma que rara vez es sentido por pacientes con cáncer al final de la vida ${ }^{(4)}$. En un estudio sobre experiencias en pacientes con cáncer avanzado y sus familias, Orreval y colaboradores mencionaron que la nutrición parenteral en casa se puede recomendar en pacientes con cáncer incurable que no pueden ser alimentados oral o por vía enteral, si se estima que morirán de inanición antes que de la progresión del tumor, debido a la obstrucción y afagia, si su desempeño y calidad de vida son aceptables y si existe una fuerte moti- vación de la familia y el paciente por demandar alguna forma de alimentación al paciente, aunque esté en la etapa terminal ${ }^{(5)}$.

Las guías ESPEN recomiendan que "en caso de falla intestinal, la nutrición parenteral prologada puede ser ofrecida si no es suficiente el aporte con nutrición oral/ enteral, si se espera que la sobrevivencia sea mayor que 2 meses y si el paciente desea continuar con esta modalidad de terapia nutricional” ${ }^{(6)}$. En el marco del cuidado paliativo, en cierto grupo de pacientes, el objetivo de la nutrición parenteral o enteral puede ser inicialmente mejorar o detener el deterioro nutricional, y asegurar una mejor sobrevivencia al paciente, mientras está en compañía de su familia. A medida que avanza la enfermedad y el manejo paliativo se centra en los síntomas, y no en el manejo de la enfermedad, la nutrición debe enfocarse en la calidad de vida y el confort de los pacientes $^{(3)}$.

En pacientes con cáncer incurable, las indicaciones para nutrición parenteral en casa representan un motivo de controversia y debate ${ }^{(3)}$. Se ha enfatizado en las características simbólicas de la alimentación en este grupo de pacientes, que tradicionalmente se ha visto como una expresión de amor y cuidado, y sin duda las familias y los cuidadores miran esto como un acto de humanidad ${ }^{(7)}$. La posición de la iglesia católica sobre el uso de nutrición e hidratación al final de la vida dice que "debe haber una presunción a favor de proveer nutrición e hidratación a todos los pacientes que requieren asistencia médica nutricional e hidratación, esto debe asegurarse si los beneficios superan los riesgos" $^{\prime(8)}$. Es importante recalcar que la terapia nutricional es un tratamiento médico que tiene unas indicaciones claras, unos riesgos y beneficios, y que necesita del consentimiento del paciente.

Esta doble forma de entender la terapia nutricional, como un cuidado básico y como una terapia médica, conlleva al médico, y demás equipo de salud, a tener conflictos éticos en cuanto a la toma de decisiones. Es por esto por lo que en este artículo presentamos el caso de un paciente de 27 años con adenocarcinoma gástrico irresecable, que lo imposibilita a tolerar una alimentación oral y enteral, y transcurre los últimos meses de 
su vida con nutrición parenteral, la cual, de haber programas de nutrición domiciliaria en las instituciones de salud del país, hubiera podido recibir en casa, y pasar los últimos días de su vida en una situación de mayor confort junto a su familia.

\section{PRESENTACIÓN DE CASO}

Paciente masculino de 27 años, quien consulta por cuadro de acidez y reflujo que se alivia al comer. Se decide ingreso para efectuar estudios complementarios. Entre los hallazgos se evidencia la presencia de líquido ascítico, que abarca los cuatro cuadrantes del abdomen, adenopatía reactiva leve inflamatoria y cambios inflamatorios de colon, recto sigmoides y de la cámara gástrica, engrosamiento moderado del fundus y cuerpo gástrico por tomografía. Se programa endoscopia, en la cual se documenta $H$. pylori positivo y lesión proliferativa a nivel del fondo gástrico, unión esófago gástrico. Se toman biopsias para realizar el diagnóstico.

Historia de apendicectomía hace 23 años. Examen físico; abdomen: ruidos gastrointestinales, aumentados en frecuencia e intensidad; abdomen blando, leve dolor a la palpación. Se reporta pérdida de 15 libras de peso en 1 mes. Se realiza tamizaje nutricional, por medio de la herramienta Nutrition Risk Score, 2002 (NRS, 2002), que indica riesgo nutricional. Se interconsulta a Nutrición, quien realiza evaluación global subjetiva (EGS), la cual revela un diagnóstico de malnutrición severa.

\section{ABORDAJE NUTRICIONAL}

El paciente presenta pérdida de peso del $11 \%$. Peso usual 146 libras; peso actual 131 libras; índice de masa corporal (IMC) $19,9 \mathrm{~kg} / \mathrm{m}^{2}$. Falta de apetito, saciedad precoz, dificultad de deglución. Se planifica dieta modificada en textura con un aporte calórico de $30 \mathrm{kcal} / \mathrm{kg} /$ día y $1,3 \mathrm{~g} / \mathrm{kg} /$ día de proteína. Se inicia suplementación oral la cual cubre el $35 \%$ de los requerimientos nutricionales. Se monitoriza la tolerancia y progresión por riesgo de síndrome de realimentación. Se recomienda control de ingesta y monitorización de tolerancia a la fórmula.

La biopsia gástrica reporta adenocarcinoma en células de anillo de sello. El paciente se lleva a cirugía, donde se encontró carcinomatosis peritoneal, y se decreta irresecabilidad de neoplasia. Se realiza una junta médica con los departamentos involucrados: oncología, cirugía, radiografía, nutrición y gastroenterología. Se concluye que el paciente cumple con los criterios de irresecabilidad por metástasis a bazo e hígado, más líquido peritoneal. Se propone manejo paliativo y quimioterapia. Egresa 15 días después con plan educacional y seguimiento por nutrición.

Reconsulta nuevamente 4 días luego de su egreso por dolor abdominal difuso, abdomen tenso, no irritación peritoneal con ascitis franca. Se procede a drenar 5000 $\mathrm{mL}$ de líquido ascítico. Luego se egresa con indicaciones para casa. A los 4 meses, el paciente consulta nuevamente a oncología para exámenes de seguimiento, habiendo recibido 4 ciclos de quimioterapia. En hallazgos de endoscopia se evidencia reducción del $50 \%$ de las lesiones tumorales gástricas. No asiste a sus citas de nutrición programadas. Sin embargo, se evalúa en oncología y luce mejorado, buen apetito, y ha recuperado el $50 \%$ del peso perdido. Tres meses después consulta a oncología para su último ciclo de quimioterapia, con Karnofsky del $90 \%$, continúa con buena evolución. No se presenta a su seguimiento a la clínica de nutrición.

Reingresa 1 mes y medio después, con historia de fiebre, dolor abdominal y vómitos con contenido biliar. La tomografía abdominal evidencia íleo espástico, engrosamiento difuso del intestino delgado y ascitis. Alta sospecha de obstrucción. Se coloca sonda nasogástrica a gravedad, y se inicia nutrición parenteral vía periférica. Es evaluado por psiquiatría, quien indica que el paciente cursa con trastorno adaptativo, mixto, depresivo.

El paciente se ha deteriorado debido a la patología de base, y no ha asistido a su seguimiento nutricional. Actualmente con 133 libras. Se inicia nutrición parenteral periférica, con un aporte inicial de $1000 \mathrm{kcal} / 1440 \mathrm{cc}$, y luego se progresa a $1400 \mathrm{kcal} / 1920 \mathrm{cc}$. A los 6 días se inicia prueba de tolerancia oral para iniciar traslape alimentación parenteral/oral. Actualmente, pesa 124 libras. Se documenta progresión de la lesión primaria y extensión del tumor al intestino delgado. El paciente tolera dieta blanda, se omite la nutrición parenteral, y luego se egresa por mejoría clínica, tolerancia a dieta oral y resolución de íleo.

A los 5 días reingresa nuevamente con datos de obstrucción, malestar general, decaimiento, condiciones generales regulares, palidez generalizada, mucosas secas y un peso de 113 libras. Se inicia nuevamente terapia con nutrición parenteral. Se coloca catéter venoso central para alimentación parenteral total a $30 \mathrm{kcal} / \mathrm{kg}$, $1,3 \mathrm{~g}$ proteína, $1 \mathrm{~g} / \mathrm{kg} /$ día de emulsiones lipídicas alternativas, $3,02 \mathrm{mg} / \mathrm{kg} / \mathrm{min}$ de dextrosa; requerimientos basales de electrolitos; ampollas de vitaminas y elementos traza. La nutrición parenteral se cicla por 18 horas, y descansa 6 horas, debido a que presenta alteración de 
pruebas hepáticas, previo al inicio de nutrición parenteral. Se monitoriza la progresión de alimentación por el riesgo de síndrome de realimentación.

El paciente presenta fiebre, y se sospecha de proceso infeccioso, para lo cual recibe cobertura antimicrobiana. Por presencia de bilirrubinas en orina y urobilinógeno se sospecha de proceso obstructivo, ya que tiene historia de metástasis hepática.

Se considera que, por crecimiento de tumor, el paciente no resuelve el cuadro de obstrucción. Se coloca sonda nasogástrica a gravedad. Continua con náuseas y vómitos, pero presenta deseo de consumir líquidos fríos y helado vía oral, lo cual se le proporciona, ya que persiste con la sonda a gravedad. Se sugiere a gastroenterología valorar la colocación de yeyunostomía, quien indica que no es factible debido a íleo, ascitis y metástasis a intestino. Se sugiere enteroscopia anterógrada para documentar si cursa con obstrucción intestinal mecánica o extrínseca de origen metastásico y, de acuerdo con esto, valorar colocación de stent.

El paciente luce caquéctico, aunque hidratado y consciente. Por persistir con alteración de pruebas de función hepática, las cuales se consideraron de origen primario metastásico, se plantea al paciente el riesgo/ beneficio de continuar con la nutrición parenteral total, a lo cual él accede a continuar dicha terapia. En junta médica se explica al paciente y a la familia su estado reservado y patología base avanzada. Se efectúa procedimiento de enteroscopia anterógrada, que documenta etiología de obstrucción incierta, y compromete la luz, por lo que no se puede colocar stent, y debe continuar con alimentación parenteral. Dos meses después de su ingreso, el paciente amanece con apetito y desea probar alimentos, los cuales se le brindan.

A los 11 meses del diagnóstico inicial, el paciente presenta falla ventilatoria. Se explica a la familia el mal pronóstico y, a petición de ellos, se realiza intubación orotraqueal, a pesar del plan educacional que se les había brindado previamente por parte de los médicos. El paciente fallece en el transcurso del día.

\section{DISCUSIÓN}

Este caso se presentó en un hospital de tercer nivel de la ciudad de Guatemala. En los pacientes con cáncer es necesario realizar una valoración nutricional temprana, identificando los factores asociados del riesgo nutricional, para así establecer estrategias eficaces de tratamiento y evitar un mayor deterioro ${ }^{(9)}$. En este caso, la primera evaluación nutricional del paciente presentó un diagnóstico de malnutrición severa, según evaluación global subjetiva, y caquexia, según los parámetros internacionales, como pérdida de peso mayor del $5 \%$, anorexia, inflamación sistémica, índice de masa corporal menor de $20 \mathrm{~kg} / \mathrm{m}^{2(10)}$. Se inició con la terapia nutricional; sin embargo, el paciente presentaba muy poca adherencia y no regresó a sus consultas.

Una vez el cáncer recidivó, ya el paciente presentaba una caquexia refractaria, y debido a la intolerancia a la dieta oral y enteral, había indicación de alimentarlo con nutrición parenteral. Esta continuó hasta el último de sus días. El paciente tenía deseos de ciertos alimentos, y como parte del cuidado paliativo, se le brindaba gelatinas o helados, los cuales regresaban al balón de la sonda a gravedad que tenía, debido a la obstrucción que padecía.

Dado a la edad, el desgaste emocional de la enfermedad y, aunado a esto, que se casaría en esos meses, el paciente presentaba un estado depresivo, diagnosticado por psiquiatría. Esto hacía que quisiera muchas veces irse a casa, y pidió en algún momento el egreso contraindicado, pero la misma obstrucción y poca tolerancia hacía que volviera a consultar. El paciente se aferraba a la vida y, al consultar, en el hospital se le brindaba la atención solicitada. Se le explicó el riesgo/beneficio de continuar con nutrición parenteral, y el paciente firmó un documento médico legal, aceptando continuar con la terapia. En Guatemala, las instituciones no cuentan con programas de alimentación parenteral en casa, por lo que esto debe contratarse de manera privada. Este era el caso de esta institución, a pesar de ser un hospital de tercer nivel. El costo de la alimentación parenteral en casa es sumamente alto y difícil de costear, mientras que en el hospital todo esto era cubierto, por lo que regresaban por la atención.

La nutrición parenteral en casa se puede recomendar en pacientes con cáncer incurable que no pueden ser alimentados de manera oral o enteral. En caso de pacientes con falla intestinal, la nutrición parenteral a largo plazo se puede ofrecer si el paciente desea continuar con esta modalidad de terapia nutricional ${ }^{(6)}$.

Se realizaron varias juntas médicas en las que se explicó al paciente y la familia la condición clínica y el mal pronóstico por la enfermedad avanzada. El paciente quería vivir, sin embargo, entendía su condición y mal pronóstico. La nutrición parenteral continuó hasta el último día de su vida debido a la obstrucción y al fuerte deseo del paciente, su prometida y su familia de aferrarse a la vida. 


\section{CONCLUSIÓN}

En este caso, el manejo nutricional se realizó tomando en cuenta su condición clínica, e individualizando cada etapa y respetando la autonomía del paciente, el cual decidió recibir nutrición parenteral hasta su última fase. Es importante establecer programas de nutrición parenteral en casa, ya que la mayoría de las instituciones hospitalarias guatemaltecas no cuentan con estos programas, y esto lo debe costear el paciente.

\section{Financiamiento}

El presente estudio no tuvo financiación.

\section{Declaración de conflictos de interés}

Sin conflictos de interés por declarar.

\section{Declaración de autoría}

Todos los autores han participado en la concepción y realización del trabajo, que ha dado como resultado el artículo en cuestión. CPMM y LDGP participaron en el análisis de los resultados; MSCL intervino en la redacción del trabajo. Todos los autores revisaron el artículo y validaron su versión final.

\section{Referencias bibliográficas}

1. Las cifras del cáncer en España 2020 [Internet]. Sociedad Española de Oncología Médica (SEOM). 2020. (Consultado el 1 de octubre 2020). Disponible en: https://seom.org/ seomcms/images/stories/recursos/Cifras_del_cancer_2020.pdf
2. Smyth EC, Verheij M, Allum W, Cunningham D, Cervantes A, Arnold D, et al. Gastric cancer: ESMO clinical practice guidelines for diagnosis, treatment and follow-up. Ann Oncol. 2016p;27(5):v38-v49. doi: 10.1093/annonc/mdw350.

3. Bozzetti F. Nutritional support of the oncology patient. Crit Rev Oncol Hematol. 2013;87(2):172-200. doi: 10.1016/j. critrevonc.2013.03.006.

4. Arends J, Bodoky G, Bozzetti F. ESPEN guidelines on enteral nutrition: non-surgical oncology. Clin Nutr. 2006;25(2):24559. doi: https://doi.org/10.1016/j.clnu.2006.01.020.

5. OrrevalY, Tishelman C, Permert J. Home parenteral nutrition: a qualitative interview study of the experiences of advanced cancer patients and their families. Clin Nutr. 2005;24:961-70. doi: https://doi.org/10.1016/j.clnu.2009.04.011.

6. Bozzetti F, Arends J, Lundholm K, Micklewright A, Zurcher G, Muscaritoli M. ESPEN guidelines on parenteral nutrition: non-surgical oncology. Clin Nutr. 2009;28(4):445-54. doi: https://doi.org/10.1016/j.clnu.2009.04.011.

7. Miles SH. Nourishment and the ethics of lament. Linacre Quarterly 1989;56(3):64-9. https://doi.org/10.1080/00243 639.1989.11878020.

8. Wildes K. A memo from the central office: the "ethical and religious directives for catholic health care services. Kennedy Inst Ethics J. 1995;5(2):133-9. doi: 10.1353/ken.0.0122.

9. Jager-Wittenaar H, Dijkstra PU, Dijkstra G, Bijzet J, Langendijk JA, van der Laan BFAM, et al. High prevalence of cachexia in newly diagnosed head and neck cancer patients: an exploratory study. Nutrition. 2017;35:114-8. doi: 10.1016/j. nut.2016.11.008.

10. Fearon KC. Cancer cachexia: developing multimodal therapy for a multidimensional problem. Eur J Cancer. 2008;44:112432. doi: https://doi.org/10.1016/j.ejca.2008.02.033. 